\title{
Towards Tool Support for the Configuration of Non-Functional Properties in SPLs
}

\author{
Julio Sincero, Wolfgang Schröder-Preikschat \\ Friedrich-Alexander University Erlangen-Nuremberg \\ $\{$ sincero,wosch $\} @$ cs.fau.de
}

\author{
Olaf Spinczyk \\ Dortmund University of Technology \\ olaf.spinczyk@tu-dortmund.de
}

\begin{abstract}
The configuration of NFPs (non-functional properties) is a crucial problem in the development of software-intensive systems. Most of the approaches currently available tackle this problem during software design. However, at this stage, NFPs cannot be properly predicted. As a solution for this problem we present the new extensions of the Feedback approach which aims at improving the configuration of NFPs in SPLs. We introduce our set of tools that are used to support the approach and show how to use them by applying it to the well-known SPL (The Graph Product Line) that was suggested as a platform for evaluating SPL technologies.
\end{abstract}

\section{INTRODUCTION}

\section{A. Software Product Lines}

The development of software product lines (SPLs) are considered [31] to be a new paradigm, as the result of its implementation, different members of a family of systems (product portfolio) can be generated. A SPL is, normally, comprised of a set of assets (software components, documentation, etc.) that can be assembled together to deliver products that satisfy the needs of a particular domain.

Several methodologies have been proposed in order to support the development of SPLs. Domain engineering [11] is the act of studying and documenting a specific domain in order to support the subsequent development phases (e.g. application engineering). Feature modeling is the approach used to capture the common, the variable and the interdependency's present in a specific domain, the results are captured in a model (normally a represented in the form of a feature diagram) that determines which sets of features are valid product specifications. Hence, feature modeling is specially important during domain engineering and also during product configuration. Several feature implementation techniques have also been proposed in order to facilitate the actual implementation of the software assets of a SPL [27], [20], [15]. Moreover, techniques for scoping [19], testing [30], managing variability [17], are also available, business aspects like risk management [31], management and financial controls [29] are also addressed. The use of these, and, of course, other methodologies, in the context of SPL development, aim at reduced time to market, reduced costs, higher productivity, better product quality [31], etc.

\section{B. Non-Functional Properties}

We see non-functional properties (NFPs) as those that do not express what a piece of software is able to compute, but how, or in which circumstances it achieves its main goals (functional properties). Examples are: code size, memory footprint, performance, security, reliability, scalability, etc. Our experience developing families of operating systems show that these properties emerge from the complex interactions among the different modules that comprise the entire system [26], [25].

\section{NFPs and SPLS}

The idea to address the configuration of NFPs in the context of SPLs is, mainly, due to two reasons (1) NFPs are very much dependent on the components that comprise the product (2) SPLs are, normally, responsible for generating the components (choosing and configuring) that are required (through a product specification) to be present in the final product. We aim at taking advantage of the infrastructure that supports product generation in SPLs to improve the process of product configuration, specially, the configuration of NFPs.

\section{Objective}

The objective of this work is to present the extensions of the Feedback approach [36] which aims at improving the configuration of NFPs in SPLs. We introduce the new set of tools that are used to support the approach, and we show how to use them by applying it to a well-known [28] SPL that was suggested as a platform for evaluating SPL technologies. Finally, we evaluate the approach and discuss about applicability.

\section{E. Outline}

The remainder of this document is organized as follows. In the next section we start with the motivation by describing advantages of addressing the configuration of NFPs in SPLs. Subsequently, we present the related work. Section IV presents the basic concepts of the Feedback approach and introduces the new set of tools that are used to implement it. A case study is presented in Section VI. The final section summarizes and concludes the work.

\section{Motivation}

Researchers have proposed many [9], [37], [2] approaches that integrate information regarding NFPs into the software development process. These techniques range from extensions of UML [9] diagrams with non-functional information up to 
the design of software architectures that take into consideration the desired non-functional behavior.

However, these techniques take into account only information gathered during requirements engineering. Although we believe these approaches improve considerably the conformity of the resulting software with the non-functional requirements, we think that a significant amount information that can improve the configuration of NFPs is simply disregarded. Additionally, as mentioned above, we believe that NFPs are heavily influenced by the composition and implementation of the different software components that make up the final software product, and it cannot be precisely predicted during software design.

Therefore, our idea is to use the information captured both at product generation (e.g. compile-time, etc.) and at runtime to use it in the configuration process to enable the explicit configuration of NFPs.

Our vision is that during product configuration (feature selection) the application engineer should be informed about the impact of each feature on the NFPs of the final product.

\section{RELATED WORK}

Our work is comprised of pieces from three major fields. First, the configuration of NFP in SPLs. Second, tool support for the SPL development. Third, reasoning in feature models. The following sections present prominent work in these areas.

\section{A. NFPs and SPLs}

Norbert et. al. [34], [32] proposes the use of a semiautomated derivation (SAD) to assist developers selecting product features in SPLs with a large number of features. The basic idea is to hide variation points that are irrelevant due to non-functional requirements that should be met. They claim that traditional approaches do not consider non-functional properties, nor alternatives for a feature implementation. To solve this problem, they present a integrated software product line model (ISPLM) which integrates code units and their nonfunctional properties into the feature model.

Benavides et. al. [4] propose an extension to feature models (as proposed by Czarnecki [10]) to accommodate information about extra-functional features (NFPs, in our view). In this approach, attributes like price or development time can be assigned to features. The features and their attributes are transformed to a constraint satisfaction problem (CSP) so that an automated reasoning can be applied to it. Hence, Optimum products can be generated according to a determined criterion (the extra-functional features).

These methods take advantage of information about NFPs to improve product configuration. However, we think that both are more related to the field of variability management [33], [24]. They ease the configuration process in large SPLs but do not offer the ability to explicit configure NFPs or to inform the user about the real influence of a feature on the required NFP.

\section{B. Tool Support}

Pure::variants [7] is a tool (Eclipse plugin) that supports variant management of SPLs. It is independent of programming languages and it enables the definition of the problem domain by means of feature models, and the solution domain by family models. It also automates the process of product generation. Regarding the configuration of NFPs, the current version is able to assign bugs (from a bug-tracking system) to specific features, therefore, during product configuration, the application engineer is informed about the known bugs that will be present on the final product.

GEARS [23] is a tool and framework that enables the development and evolution of SPLs, it applies the 3-tiered SPL methodology [22]. In GEARS, SPLs are comprised of three elements, Software assets (source code, documentation, etc.), product feature profile (to model each product in the portfolio), and the gears configurator which automatically assembles products based on its specification.

FeaturePlugin [1] is an open-source Eclipse plugin for designing and configuring feature models. It supports the concepts of staged configuration [13] and feature cardinalities [12]. The tool focus on providing advanced techniques of feature modeling and not in supporting the whole process of SPL development.

FAMA (FeAture Model Analyser) [5] is an extensible framework for the automated analysis of feature models. It is able to denote feature models in several logic representations, therefore, different solvers can used in the analysis process. Currently, it supports CSP, SAT (boolean satisfiability problem) and BDD (binary decision diagrams), but it is flexible enough to have other solvers added to it. Cardinality-based feature models are allowed and the following operation are supported: finding out if a feature model is valid (it exists a valid selection that satisfies all constraints), finding the total number of valid products, list all valid configurations and calculating the commonality of features (the number of valid products it appears).

There are commercial, free and open-source alternatives for the design of feature models, only pure-variants is able to provide non-functional information during product configuration, at the moment very basic, though.

\section{Reasoning in Feature Models}

Important to our work is also the process of reasoning in feature models. A seminal work of Benavides et. al. [6], presents the mapping from feature model components (e.g. optional features, mandatory feature, and group features) to diverse logical representations, namely, SAT, BDD and CSP. Transforming feature models in these representations enables the use of off-the-shelf solvers that can perform several analysis very relevant for feature models (e.g. validity, number of solutions, etc.).

The relation of feature models and grammars have also been studied [3]. Batory, also motivated by the ability to use off-the-shelf satisfiability solvers, presented the mapping from feature models, firstly, to iterative tree grammars, and then 
to propositional formulas. However, his main goal was to simplify the laborious task of debugging feature models.

Recently, the relation of feature models and logic representation have been further explored. Czarnecki et. al. [14] proposes a method for the inverse transformation, from propositional formulas to feature model representation. Janota et. al. [18] studies the representation of feature models in higherorder logic, a formalized meta-model is presented, however, no tool support is provided.

\section{THE Feedback APPROACH}

The Feedback approach extends the traditional SPL development techniques in order to provide information regarding NFPs during product configuration. We introduced new structures and mechanisms so that the SPL infrastructure can be used to generate products that will be tested against the desired NFP. This information is saved, organized, and reinserted in the SPL, it enables the user to benefit from it on the configuration of further products.

This process is organized in three layers:

SPL Repository is comprised of the software components that can be assembled together to generate products. Additionally, components that are used merely to capture non-functional information from generated products are also available. We have shown [16] that aspects are very adequate for this task.

User Configuration is responsible for providing the mechanisms for product configuration. Besides the traditional configuration process by selecting features from a feature model, we provide the user with non-functional information. As NFPs are very specific to each product, or even features, this information can be displayed in different ways, for example, sliders, graphs, charts, etc. Moreover, during configuration the user can select the aforementioned components that are responsible for checking the NFPs of the product.

Concrete Solution Domain encompasses the generated product and the compile/runtime environment used to generate and test the product.

The organization of the SPL in this fashion aims at improving the configuration experience in the following ways: (i) providing exact information about products that have been previously configured. (ii) using heuristics and regression techniques to provide approximated information about products that have been only partially configured. (iii) if more then one software component implement the same functional behavior, the user should be able to select the most appropriate by means of its non-functional characteristics.

\section{TOOL-CHAIN ARCHITECTURE}

This paper introduces our new set of tools that support the implementation of the Feedback approach. Basically, it consists of a feature modeling tool, a logic solver and a set of scripts. Figure 1 depicts the whole process and the tools involved, details are explained in the following sections.

\section{A. Turning the LKC into a Feature Modeling Tool}

Our approach is centered on feature modeling. However, our initial goal was not to develop a feature modeling tool from scratch, but to integrate the Feedback approach into an existing tool. The only feature modeling tool that, to the best of our knowledge, provides freely its source code is the FeaturePlugin [1]. Nevertheless, it requires the whole Eclipse project, as we did not want to force the use of Eclipse to deploy our approach, we had to turn this option down. Later, our work analyzing the Linux Kernel [35] as a SPL has shown that its configuration tool could be turned into a feature modeling tool.

The Linux Kernel Configurator (LKC) is a tool that is delivered within the Linux Kernel in order to enable its configuration (feature selection). Its first prototype was proposed in 2002, the current version is 1.3, and as the Linux Kernel, it is released under the GNU General Public License (GPL).

Table I presents the mappings that we propose for the the design of feature models using the LKC language constructs.

Most of the mappings were relatively easy to perform. For the mandatory relation, the parent feature forces the selection of the child by the use of a reverse dependency (select). The optional relation is described by using a dependency between the child and the parent feature (depends on). The or group is designed by creating reverse dependencies between the children and the parent, this was done inside a menu definition in order to group the children together. The alternative group can be described by including configuration options (the children) inside a choice definition, which has the same semantic as of alternative group in feature models. Extra constrains like implies and excludes are defined using normal and negated dependencies (requires).

Using these mappings we were able to design several feature models. So far we did not find any feature model construction that could not be modeled with the LKC language. Hence, it was chosen as the feature configuration tool for the implementation of the Feedback approach.

\section{B. Partial Configuration}

As mentioned earlier, the Feedback approach enables the user to generate products, run tests on it and save real information about NFPs to improve the configuration of further products.

In our view, the collection of non-functional information can be performed in two ways. First, generating single products and performing tests on it (left side of Figure 1). Second, generating sets of products to be tested on the same scenario (right side of Figure 1).

To generate sets of product specifications we use the concept of partial configuration, which is a feature selection where features can be selected (the feature must be present) or blocked (must not be present). Using the partial configuration we generate the set of valid product specifications that respect the selected and blocked features. This process is shown on the right side of Figure 1. Using the feature modeling tool, the user performs a partial configuration, which is the input of our 

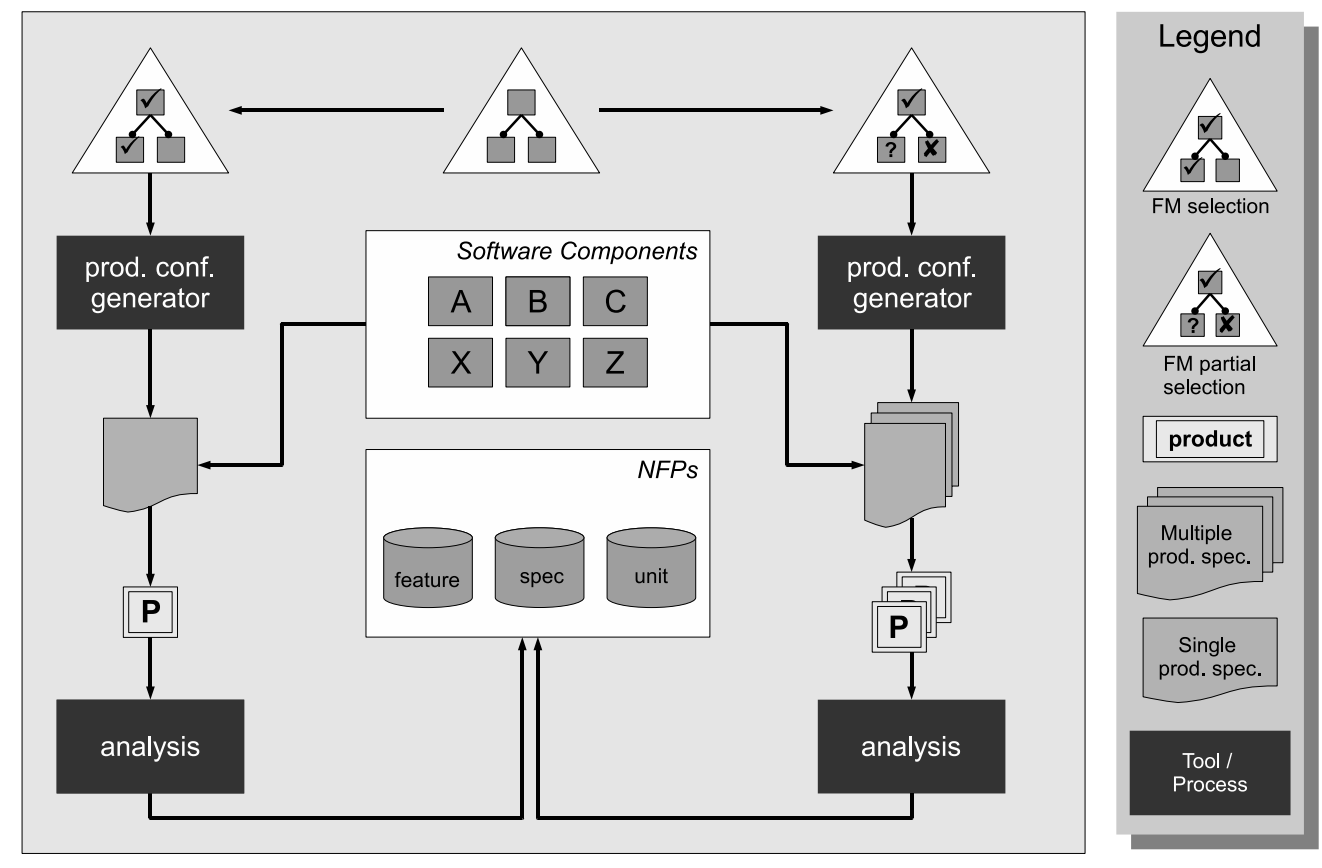

Figure 1. Overview of the Feedback Approach

generator (the feature model is translated to a binary decision diagram, using the translation as proposed by Benavides [6], and implemented using the BuDDy library [8]). The generator output is a set of specifications that are used to generate the corresponding set of products.

\section{Running Tests}

In our tool set, product specifications are simple text files with pre-processor directives (defines) to control the features that should be enabled or not. Using the specification generated as explained in the previous section, we are able to generate sets of products and run them for the evaluation of the required NFP.

As shown on the bottom part of Figure 1, the result of the product analysis can be associated to a feature (specific feature present in the model), to a spec (complete product configuration), or to a unit (implementation unit, like classes or aspects).

\section{Case Study}

In order to evaluate our approach we conducted one case study. We have chosen the Graph Product Line (GPL) [28] for several reasons, it was proposed as a platform for evaluating SPLs, the domain of graphs is well understood and the algorithms are well known, and finally, recent work [21] has used it for evaluating new techniques for implementing products lines.

\section{A. The GPL Product Line}

The GPL implements a family of classical graph applications. Its feature model is depicted in Figure 3, as it can be seen, basically, its variation points are the graph type (directed

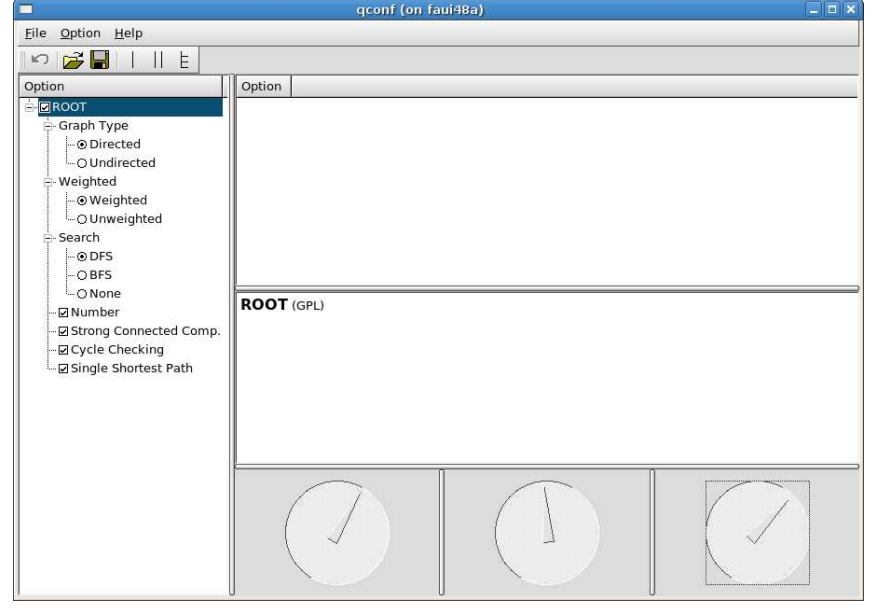

Figure 2. The extended LKC Tool as a Feature Modeling Tool

or undirected), the search algorithm (DFS or BFS) ${ }^{1}$, and edge type (weighted and unweighted). Additionally, a set of algorithms to handle graphs are also available:

Number (Number): assigns a unique number to each vertex a the result of a traversal.

Connected Components (Con.Comp.): computes the connected components of an undirected graph.

Strongly Connected Components $(S C C)$ : computes the strongly connected components of a directed graph.

Cycle Checking (CycleCheck): checks the existence of cycles in a graph.

Minimum Spanning Tree (MSTP. and MSTK): computes

\footnotetext{
${ }^{1}$ DFS=depth-first search, BFS=breadth-first search
} 
Table I

MAPPING: FEATURE RELATIONS TO LKC LANGUAGE

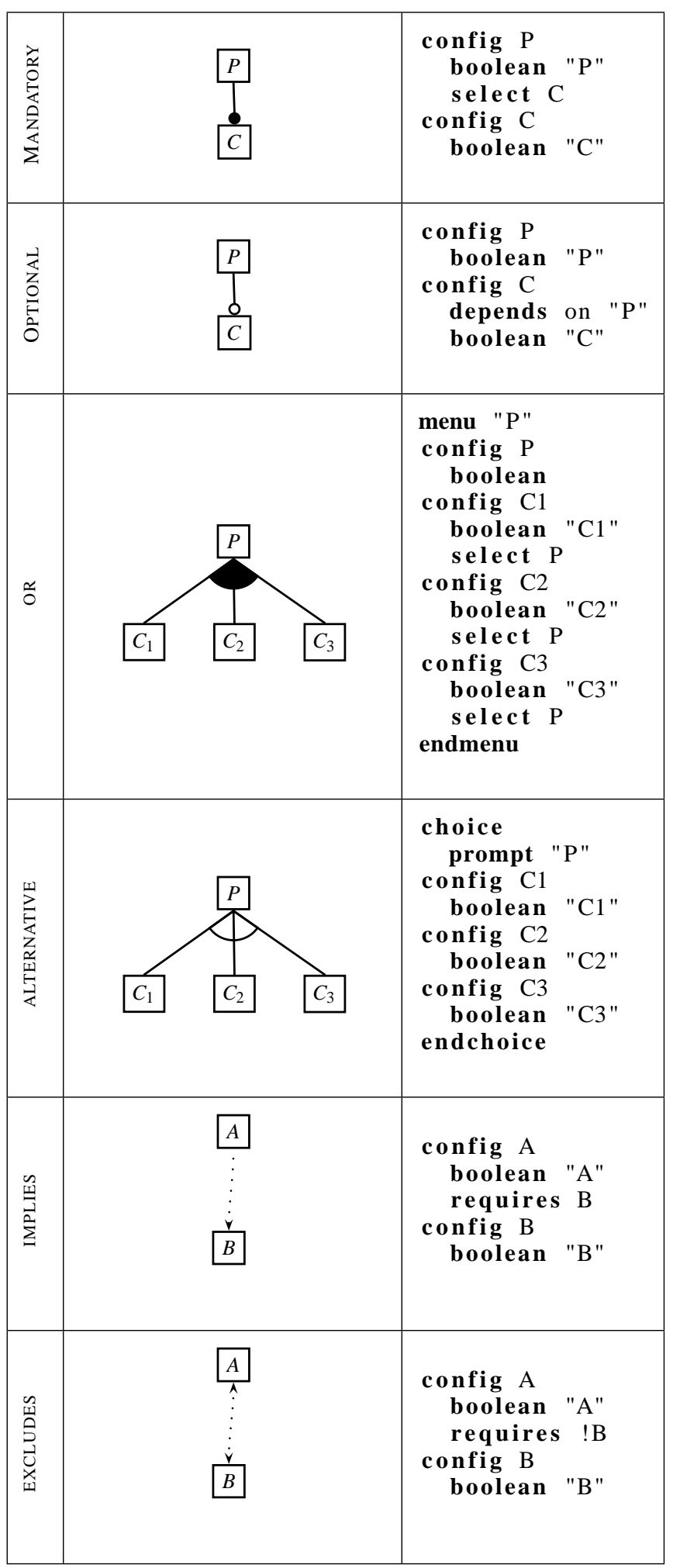

the mininum spanning tree, which is a spanning tree with weight less then or equal to the weight of every other spanning tree. Two implementations of this feature are available.

Single-Source Shortest Path $(S S P)$ : computes the shortest path from a source to all other vertices.

Table III shows the extra constraints that cannot be shown on the feature model, and, therefore, are implemented as an implies relation (shown on Table I). Each line shows which features can be associated to each algorithm.

It may seem to be a very simple feature model, however, even with only 19 features and a couple of extra constraints the idea to provide non-functional information about every of its possible valid feature combinations, is a very challenging task.

\section{B. Targeted NFPs}

So far in this document we have discussed about NFP in a very general way. This was due to the following reason: NFPs are totally dependent on scenarios and standpoints. For some stakeholders, the definition of performance or security may have completely different means than for other stakeholders. Therefore, we think that NFPs should be specifically defined. For example, the question "what is the performance of pro$\operatorname{duct} X$ ?", is way too generic, and, in our opinion, cannot be satisfactorily answered. Nevertheless, a question like "what is the time required to perform the number algorithm in a graph with 300 vertices and 45k edges?", is a very appropriate question, which shows a relevant way to represent one of the several ways that the NFP performance could be seen in this scenario. The Feedback aims at providing the visualization/configuration of NFPs defined in this manner during product configuration.

In order to test this concept, and the Feedback approach itself, we have defined the follow NFP to be tested: the performance of the number algorithm for three different workloads. That is, the time required to perform the algorithm in randomly generated graphs of the sizes: 300 vertices and $125 \mathrm{k}$ edges, 500 vertices and $45 \mathrm{k}$ edges, and, 1000 vertices and $500 \mathrm{k}$ edges. To be able to provide the information resulting from these tests during product configuration, we generated a set of variants of the GPL using the process described in Section V-B. We have set the feature number as selected and all other features under algorithm as blocked. Our generator output 8 different product configurations that were tested using the three different workloads. The results are shown on Table II (time in milliseconds). Our feature modeling tool shown in Figure 2 displays this information during feature selection. As it can be seen on the bottom of Figure 2, we are using rounded range control components (one for each workload) to display the performance (as defined above) of the current selection.

This process enables us to display exact information about NFPs that were specifically defined and tested. We believe that this information helps the user on the process of deciding which features should be included or not, or, at least, informing 
Table II

NUMBER TESTS

\begin{tabular}{|c||c|c|c|}
\hline Conf. & Workload1 & Worload2 & Workload3 \\
\hline 1 & 110 & 378 & 2868 \\
\hline 2 & 111 & 380 & 2876 \\
\hline 3 & 153 & 501 & 3298 \\
\hline 4 & 161 & 493 & 3361 \\
\hline 5 & 109 & 385 & 2848 \\
\hline 6 & 111 & 377 & 2856 \\
\hline 7 & 157 & 496 & 3340 \\
\hline 8 & 153 & 513 & 3358 \\
\hline
\end{tabular}

about the non-functional behavior that should be expected in the final product.

\section{CONClusions AND Future WORK}

There are many ways to address the configuration of NFPs of a system. Most of the approaches annotates models during software design with non-functional information/requirements that are of interest. However, the real behavior of a piece of software can be verified only at runtime and when deployed in real scenarios.

To tackle this problem we devised the Feedback approach. We developed mechanisms to generate sets of products so that desired NFPs can be tested in a real scenario. Additionally, this information can be saved for further reuse. The application engineer using our configuration tool is able to see what is the impact of feature selection on NFPs that were previously tested.

In our case study we have shown the feasibility of the idea, and also, that with appropriate tool support it is relatively easy to implement.

In order to extend and further evaluate out work, we plan to improve the integration of our tools and to carry out a case study in a large scale SPL with thousands of features. The analysis of other types of NFPs (security, latency, etc.) in operating systems are also under work. Moreover, we are also investigating the inverse way to configure a product, enabling the user to set constraints regarding the tested NFPs, and the configuration tool will generate the sets of products that are able to fulfill the requirements. Regarding selections that were not tested, we are applying regression techniques to predict its behavior.

\section{REFERENCES}

[1] Michal Antkiewicz and Krzysztof Czarnecki. FeaturePlugin: feature modeling plug-in for Eclipse. In 2004 OOPSLA workshop on Eclipse technology eXchange (Eclipse '04 at OOPPSLA '04), pages 67-72, Vancouver, Canada, July 2004.

[2] Len Bass. Principles for designing software architecture to achieve quality attribute requirements. In SERA '06: Proceedings of the Fourth International Conference on Software Engineering Research, Management and Applications, page 2, Washington, DC, USA, 2006. IEEE Computer Society.

[3] Don S. Batory. Feature models, grammars, and propositional formulas. In 9th Software Product Line Conf. (SPLC '05), pages 7-20, 2005.

[4] D. Benavides, A. Ruiz-Cortés, and P. Trinidad. Automated reasoning on feature models. LNCS, Advanced Information Systems Engineering: 17th International Conference, CAiSE 2005, 3520:491-503, 2005.
[5] D. Benavides, S. Segura, P. Trinidad, and A. Ruiz-Cortés. FAMA: Tooling a framework for the automated analysis of feature models. In Proceeding of the First International Workshop on Variability Modeling of Software-Intensive Systems (VAMOS), 2007.

[6] D. Benavides, S. Segura, P. Trinidad, and A. Ruiz-CortÃ@s. A first step towards a framework for the automated analysis of feature models. In Managing Variability for Software Product Lines: Working With Variability Mechanisms, 2006.

[7] Danilo Beuche. Variant management with pure::variants. Technical report, pure-systems GmbH, 2003. http://www.pure-systems.com/.

[8] BuDDy Project. http://sourceforge.net/projects/buddy.

[9] Luiz Marcio Cysneiros and Julio Cesar Sampaio do Prado Leite. Nonfunctional requirements: From elicitation to conceptual models. IEEE Transactions on Software Engineering, 30(5):328-350, 2004.

[10] Krysztof Czarnecki and Ulrich W. Eisenecker. Generative Programming. Methods, Tools and Applications. AW, May 2000.

[11] Krzysztof Czarnecki. Generative Programming: Principles and Techniques of Software Engineering Based on Automated Configuration and Fragment-Based Component Models. PhD thesis, Technische Universität Ilmenau, Ilmenau, Germany, 1998.

[12] Krzysztof Czarnecki, Simon Helsen, and Ulrich W. Eisenecker. Formalizing cardinality-based feature models and their specialization. Software Process: Improvement and Practice, 10(1):7-29, 2005.

[13] Krzysztof Czarnecki, Simon Helsen, and Ulrich W. Eisenecker. Staged configuration through specialization and multilevel configuration of feature models. Software Process: Improvement and Practice, 10(2):143169, 2005.

[14] Krzysztof Czarnecki and Andrzej Wasowski. Feature diagrams and logics: There and back again. In 11th Software Product Line Conf. (SPLC '07), pages 23-34, 2007.

[15] Cristina Gacek and Michalis Anastasopoules. Implementing product line variabilities. In 2001 Symp. on Software Reusability (SSR '01), pages 109-117. ACM, 2001.

[16] Wasif Gilani, Julio Sincero, and Olaf Spinczyk. Aspectizing a web server for adaptation. In Twelfth IEEE Symposium on Computers and Communications (ISCC'07), Aveiro, Portugal, 2007. IEEE.

[17] Jilles Van Gurp, Jan Bosch, and Mikael Svahnberg. On the notion of variability in software product lines. wicsa, 0:45, 2001.

[18] Mikolás Janota and Joseph Kiniry. Reasoning about feature models in higher-order logic. In 11th Software Product Line Conf. (SPLC '07), pages 13-22, 2007.

[19] I. John, J. Knodel, T. Lehner, and D. Muthig. A practical guide to product line scoping. In 10th Software Product Line Conf. (SPLC '06), pages 3-12, Aug. 2006.

[20] Christian Kästner, Sven Apel, and Don Batory. A case study implementing features using AspectJ. In 11th Software Product Line Conf. (SPLC '07), pages 223-232. IEEE, 2007.

[21] Christian Kästner, Sven Apel, and Martin Kuhlemann. Granularity in software product lines. In ICSE, pages 311-320, 2008.

[22] Charles W. Krueger. The 3-tiered methodology: Pragmatic insights from new generation software product lines. In 11th Software Product Line Conf. (SPLC '07), pages 97-106, 2007.

[23] Charles W. Krueger. BigLever software Gears and the 3-tiered SPL methodology. In OOPSLA '07: Companion to the 22nd ACM SIGPLAN conference on object-oriented programming systems and applications, pages 844-845, New York, NY, USA, 2007. ACM.

[24] Felix Loesch and Erhard Ploedereder. Optimization of variability in software product lines. In 11th Software Product Line Conf. (SPLC '07), pages 151-162, 2007.

[25] Daniel Lohmann, Wolfgang Schröder-Preikschat, and Olaf Spinczyk. Functional and non-functional properties in a family of embedded operating systems. In 10th IEEE Int. W'shop. on Object-oriented Realtime Dependable Systems (WORDS '05), pages 413-420, Sedona, AZ, USA, February 2005.

[26] Daniel Lohmann, Olaf Spinczyk, and Wolfgang Schröder-Preikschat. On the configuration of non-functional properties in operating system product lines. In 4th AOSD W'shop on Aspects, Components, and Patterns for Infrastructure Software (AOSD-ACP4IS '05), pages 19-25, Chicago, IL, USA, March 2005. Northeastern University, Boston (NUCCIS-05-03)

[27] Daniel Lohmann, Olaf Spinczyk, and Wolfgang Schröder-Preikschat. Lean and efficient system software product lines: Where aspects beat objects. In Awais Rashid and Mehmet Aksit, editors, Transactions on AOSD II, number 4242 in LNCS, pages 227-255. Springer, 2006. 


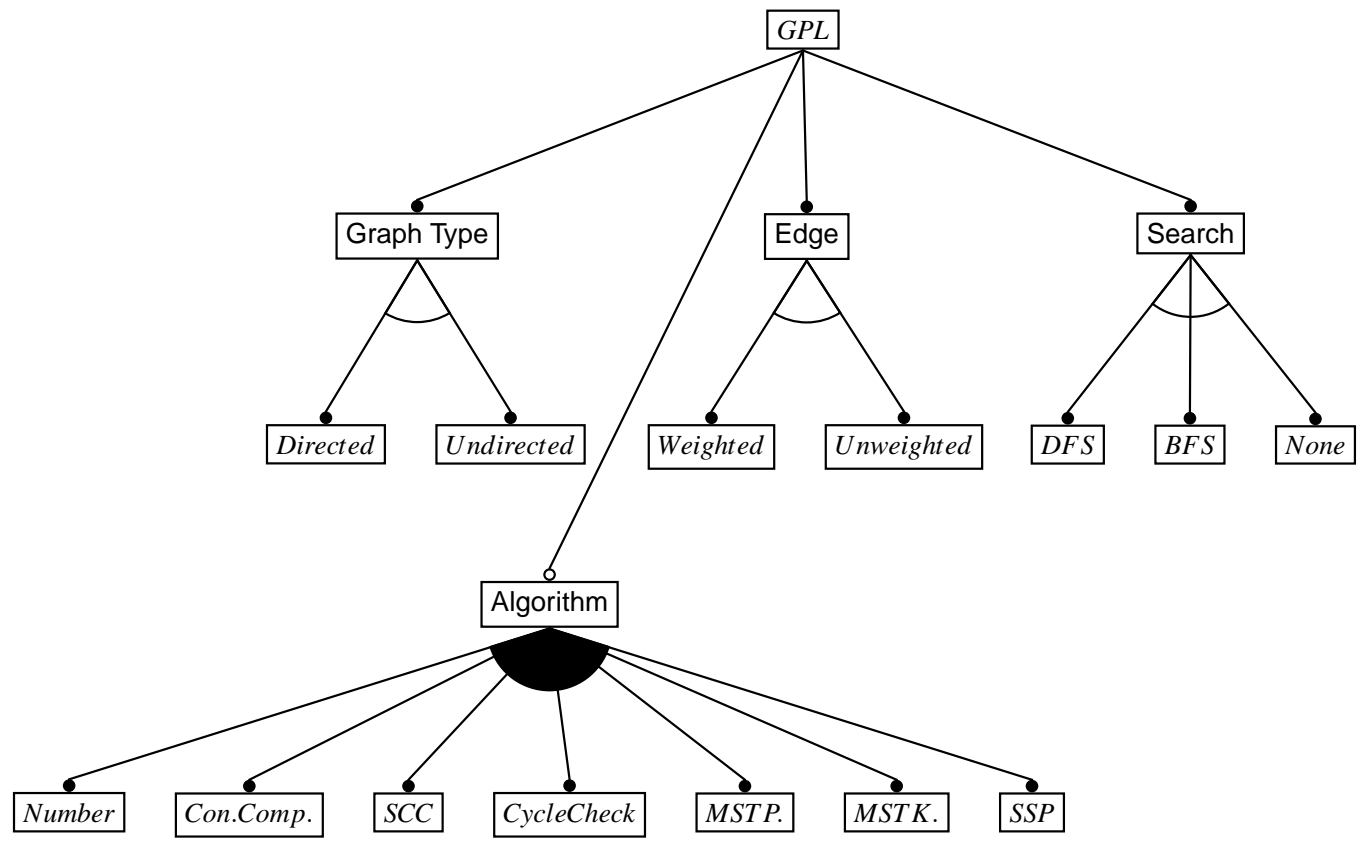

Figure 3. Graph Product Line Feature Model

Table III

GRAPH PRODUCT LINE CONSTRAINTS

\begin{tabular}{|c|c|c|c|c|c|c|c|}
\hline & \multicolumn{3}{|c|}{ Search } & \multicolumn{2}{c|}{ Graph Type } & \multicolumn{2}{c|}{ Edge Type } \\
\hline Algorithm & None & BFS & DFS & Directed & Undirected & Weighted & Unweighted \\
\hline NUMBER & & $\times$ & $\times$ & $\times$ & $\times$ & $\times$ & $\times$ \\
\hline CC & & $\times$ & $\times$ & & $\times$ & $\times$ & $\times$ \\
\hline SCC & & & $\times$ & $\times$ & & $\times$ & $\times$ \\
\hline CYCLE & & & $\times$ & $\times$ & $\times$ & $\times$ & $\times$ \\
\hline MST & $\times$ & & & & $\times$ & $\times$ & \\
\hline SSP & $\times$ & & & $\times$ & & $\times$ & \\
\hline
\end{tabular}

[28] Roberto E. Lopez-Herrejon and Don Batory. A standard problem for evaluating product-line methodologies. Lecture Notes in Computer Science, 2186:10-??, 2001.

[29] Yoshihiro Matsumoto. A guide for management and financial controls of product lines. In 11th Software Product Line Conf. (SPLC '07), pages 163-170, 2007.

[30] John D. McGregor. Building reusable testing assets for a software product line. In 10th Software Product Line Conf. (SPLC '06), page 220, 2006.

[31] Linda Northrop and Paul Clements. Software Product Lines: Practices and Patterns. AW, 2001.

[32] M. Rosenmüller, N. Siegmund, H. Schirmeier, Julio Sincero, Sven Apel, Thomas Leich, Olaf Spinczyk, and Gunter Saake. FAME-DBMS: Tailor-Made Data Management Solutions for Embedded Systems. In Workshop on Software Engineering for Tailor-Made Data Management (SETMDM), 2008.

[33] Horst Schirmeier and Olaf Spinczyk. Tailoring Infrastructure Software Product Lines by Static Application Analysis. In 11th Software Product Line Conf. (SPLC '07), pages 255-260. IEEE, 2007.

[34] N. Siegmund, M. Kuhlemann, M. Rosenmüller, C. Kästner, and G. Saake. Integrated product line model for semi-automated product derivation using non-functional properties. In International Workshop on Variability Modelling of Software-Intensive Systems (VAMOS), pages 25-23, January 2008.

[35] Julio Sincero, Horst Schirmeier, Wolfgang Schröder-Preikschat, and Olaf Spinczyk. Is The Linux Kernel a Software Product Line? In Frank van der Linden and Björn Lundell, editors, International Workshop on Open Source Software and Product Lines (SPLC-OSSPL 2007), Kyoto, Japan, 2007.

[36] Julio Sincero, Olaf Spinczyk, and Wolfgang Schröder-Preikschat. On the
Configuration of Non-Functional Properties in Software Product Lines. In 11th Software Product Line Conf., Doctoral Symp. (SPLC '07), 2007. [37] L. Xu, H. Ziv, D. Richardson, and Z. Liu. Towards modeling nonfunctional requirements in software architecture. 